\title{
Development of a Co-Deposition Method of Ceramic Particles for Hydrogen Permselective thin Pd films
}

\author{
Mikihiro Nomura $^{1 *}$, Tasuku Ono ${ }^{1}$, Akane Yamamoto $^{1}$, Tetsuaki Tsubono ${ }^{1}$ and Koichi Suzuki ${ }^{2}$ \\ 1: Department of Applied Chemistry, Shibaura Institute of Technology, 3-7-5 Toyosu, Koto-ku, Tokyo 135-8548, Japan \\ 2: Electronic Device Operations, Dai Nippon Printing Co. \\ Fax: 81-3-5859-8160, e-mail: Lscathy@shibaura-it.ac.jp
}

Pd membranes show high $\mathrm{H}_{2}$ permselectivity. In this study, ceramic particles were co-deposited in a Pd thin film to reduce Pd amounts of the thin films. Additives for ceramic particles were investigated. Pd films were prepared by using an electroplating at $0.20 \mathrm{~A} \mathrm{~cm}^{-2}$. CTAB (Cetyl trimethyl ammonium bromide: +), PEG (polyethylene glycol: \pm ), TPAOH (tetrapropyl ammonium hydroxide: + ), BTMAOH (benzyltrimethylammonium hydroxide: + ) and SDS (Sodium Dodecyl Sulfate: -) were employed for additives. Effects of charge and viscosity of the additives were tested using LTA zeolites (about $1 \mu \mathrm{m}$ ) for ceramic particles. Pd thin films were characterized by using surface and bulk EDX. LTA ratio for CTAB is much higher than those of PEG and SDS. Positive charge is effective for co-deposition, because Pd ion is also positively charged. LTA ratio for CTAB is also higher than those of TPAOH and BTMAOH. The charge of the additives must be key factor of the co-deposition. Surface ratio increased for TPAOH and BTMAOH samples. However, no relationship was found between surface ratios and the viscosity of the electrolyte.

Key words: $\mathrm{H}_{2}$ separation, thin Pd film, electroplating, ceramic co-deposition

\section{INTRODUCTION}

Thin Pd films are excellent material for $\mathrm{H}_{2}$ permselective membrane. A dense Pd membrane shows high $\mathrm{H}_{2}$ permeance and $\mathrm{H}_{2}$ permselectivity [1]. However, $\mathrm{Pd}$ is a precious metal. Thus, many research groups have been developing to reduce $\mathrm{Pd}$ amounts of $\mathrm{H}_{2}$ permselective metal membranes by using thin Pd films [1] and alloy of $\mathrm{Pd}$ and $\mathrm{Ag}$ or $\mathrm{Cu}$. Non-Pd metals such as group IVa, $\mathrm{V}$ elements (V, $\mathrm{Nb}$, etc.) [2] or $\mathrm{Zr}$ amorphous alloy [3] have been investigated.

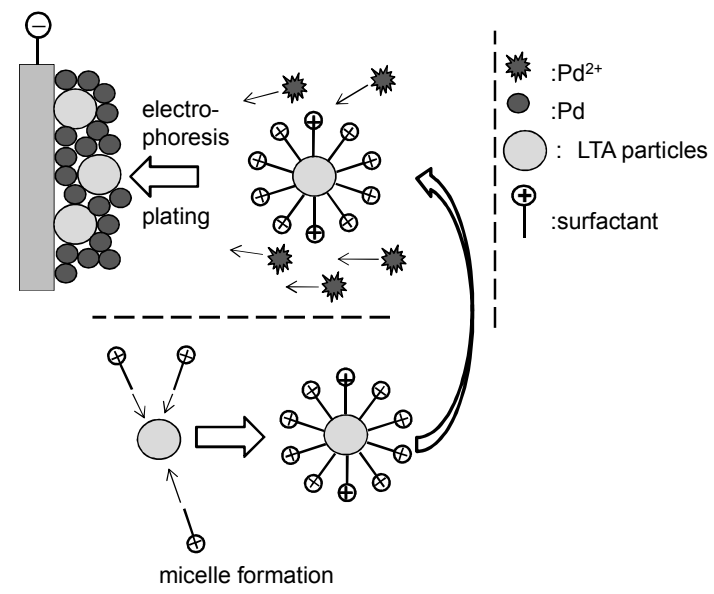

Fig.1 Schematic diagram of co-deposition mechanism of Pd and LTA particles using a surfactant as a positive charged micelle
We have been developing to prepare Pd thin films by using an electroplating. The surface morphologies of Pd films were controlled by changing the viscosity of the electroplating solutions [4, 5]. In this study, ceramic particles were co-deposited in a Pd thin film. In order to co-deposite the ceramic particles, effects of additives such as surfactants were tested. Fig. 1 shows a schematic diagram for co-deposition of $\mathrm{Pd}$ and LTA ceramic particles. The surface electric charge for usual ceramics is very low. Thus, effects of additives should be important.

\section{EXPERIMENTAL}

Dense SUS plates $\left(7 \mathrm{~cm}^{2}\right)$ were employed for substrates of the thin Pd films. SUS plates were washed by using an acid and a base before the electroplating. $\mathrm{Ni}$ strike electroplating was conducted for an intermediate layer between a SUS substrate and a Pd layer to reduce peeling off. The film thickness of $\mathrm{Pd}$ was calculated from the current efficiency ( $30 \mathrm{mg} \mathrm{A}^{-1} \mathrm{~min}^{-1}$ ) assuming that the film thickness is uniform on the substrate. The electroplating was conducted under current density of $0.20 \mathrm{~A} \mathrm{~cm}^{-2}$ for $135 \mathrm{~s}$ (thickness: $10 \mu \mathrm{m}$ ) under stirring $(0-400 \mathrm{rpm})$. LTA zeolite particles (Mizusawa Ind. Chem. Co.) were used as ceramic particles. LTA $\left(\mathrm{Na}_{12} \mathrm{Al}_{12} \mathrm{Si}_{12} \mathrm{O}_{48}\right)$ is an alminosilicate crystal with 0.5 $\mathrm{nm}$ pores. Concentration of LTA in the electroplating solution was kept at $100 \mathrm{~g} \mathrm{~L}^{-1}$. 5 types of the additives for LTA coating were employed. Cetyltrimethylammonium bromide (CTAB: Wako Pure Chem. Co.), polyethylene glycol (PEG: M.W. 20,000, Wako Pure Chem. Co.), Sodium dodecyl sulfate (SDS: Wako Pure Chem. Co.), tetrapropylammonium 
Table 1 Additives used in this article

\begin{tabular}{llcll}
\hline Additives & \multicolumn{3}{c}{ charge } & viscosity \\
\hline Cetyltrimethylammonium bromide & CTAB & + & surfactant & high \\
Polyethylene glycol & PEG & \pm & surfactant & high \\
Sodium Dodecyl Sulfate & SDS & - & surfactant & high \\
Tetrapropylammonium hydroxide & TPAOH & + & quaternary ammonium & low \\
Benzyltrimethylammonium hydroxide & BTMAOH & + & quaternary ammonium & low \\
\hline
\end{tabular}
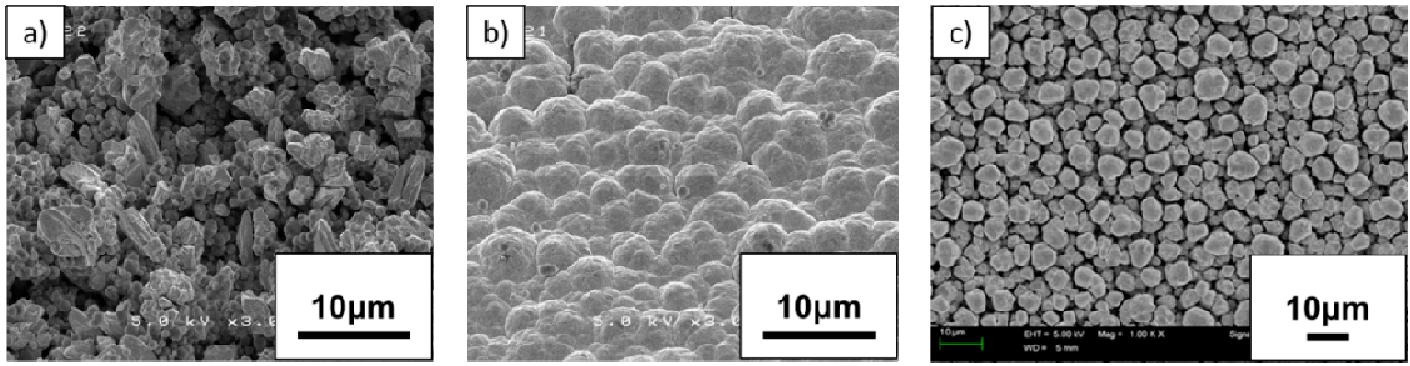

Fig. 2 SEM images of surface observation for Pd films with or without addition of LTA stirring at $400 \mathrm{rpm}$, (a) with LTA (at the edge), (b) with LTA (at the center), (c) without LTA (at the center)

hydroxide (TPAOH: Sigma-Aldrich Co.) and benzyltrimethylammonium hydroxide (BTMAOH: Sigma-Aldrich Co.) were employed for additives. The detailed information for additives is summarized in Table 1 . These additives were selected by considering charge and viscosity.

An optical microscope (LG130PC: Shodensha Inc.) and scanning electric microscope (SEM UTLRA55: Carl Zeiss Inc.) were employed for surface observations of Pd films. Atomic concentrations were measured by using an EDX. LTA concentrations were estimated by measuring Si concentrations in Pd films. Surface ratio (measured surface area/ surface area before electroplating) was measured by using an atomic force microscopy (AFM Vert Scan 2.0: Ryoka Systems Inc.).

\section{RESULTS AND DISCUSSIONS}

3.1 Electroplating without additives

First, Pd electroplating with only LTA particles was investigated. Fig. 2 shows surface SEM images for the Pd films with or without LTA particles. All the images show the rough surfaces. The surface morphologies with LTA samples ((a) and (b)) differ by the observation places. This is due to current density difference by the plating places. Usually, the current density increases at the edge of the electroplating substrate. So, the surface at the edge (a) was rough compared with the surface at the center (b). The center images are shown in the following discussions. Surface roughness slightly changed by adding LTA particles from the figure ((b) and (c)). These rough structures were called a dendric structure.

This dendric surface roughness was considered to be due to diffusion limitation of $\mathrm{Pd}$ ions during the electroplating. The balance of the rate of Pd diffusion in the solution and that of plating is important to obtain dendric structures. When Pd diffusion is relatively fast, the obtained structure is smooth. While Pd diffusion is relatively slow, the obtained structure is rough. So, the roughness increases with increasing current densities, because, Pd diffusion is the constant. On the other hand, when $\mathrm{Pd}$ diffusion is restricted by increasing solution viscosity, the surface structure is also rough. Detailed discussions about dendric structures are described elsewhere [4]. Thus, diffusion of $\mathrm{Pd}$ ions might be changed by adding LTA particles from the comparison of the images (b) and (c).

LTA particles were not found from the SEM images (a) and (b). The particle size of LTA was about $1 \mu \mathrm{m}$. Si was not found by EDX measurements from both samples indicating that LTA particles were not co-deposited by just adding LTA particles to the electroplating solutions. Effective additives are required for the co-deposition. The effects of additives are described in the next section.

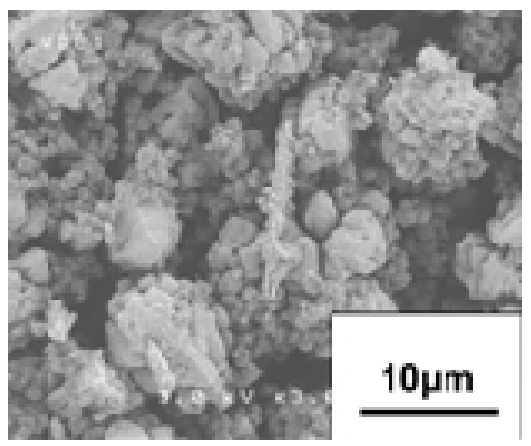

Fig.3 SEM image for the surface of the sample with CTAB $\left(1.4 \times 10^{-3} \mathrm{~mol} \mathrm{~L}^{-1}\right.$, without stirring)

\subsection{CTAB addition}

Next, CTAB was added to the electroplating solution. $\mathrm{CTAB}$ is a positive charged surfactant. Fig. 3 shows the surface image for $1.4 \times 10^{-3} \mathrm{~mol} \mathrm{~L}^{-1}$ of CTAB concentration without stirring. Critical micelle concentration (CMC) of CATB is $1.56 \times 10^{-3} \mathrm{~mol} \mathrm{~L}^{-1}$. 

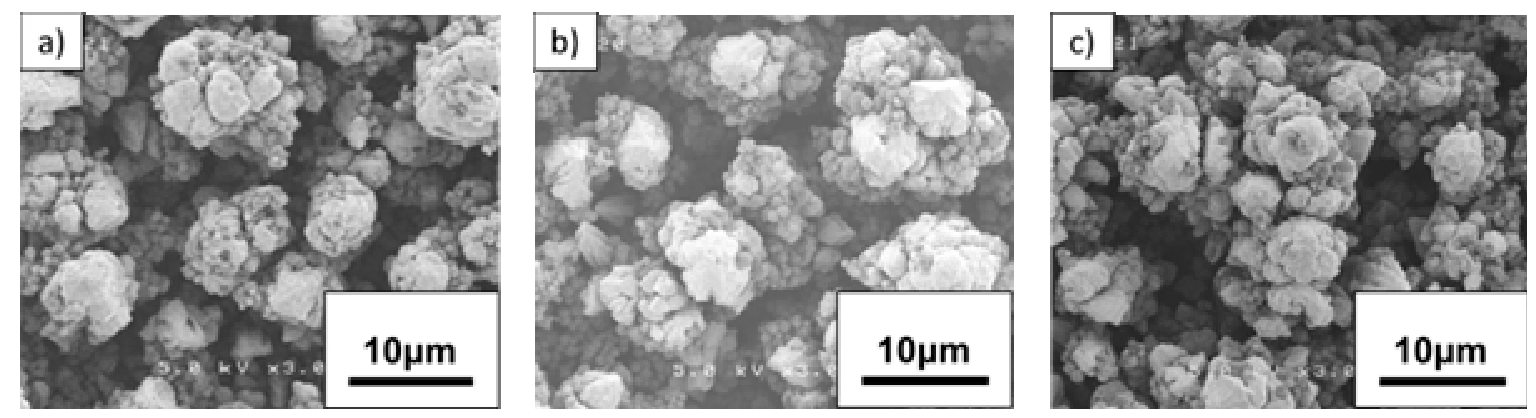

Fig.4 SEM images of surface observation for Pd films with or without addition of LTA without stirring, (a) with PEG, (b) with TPAOH, (c) with BTMAOH

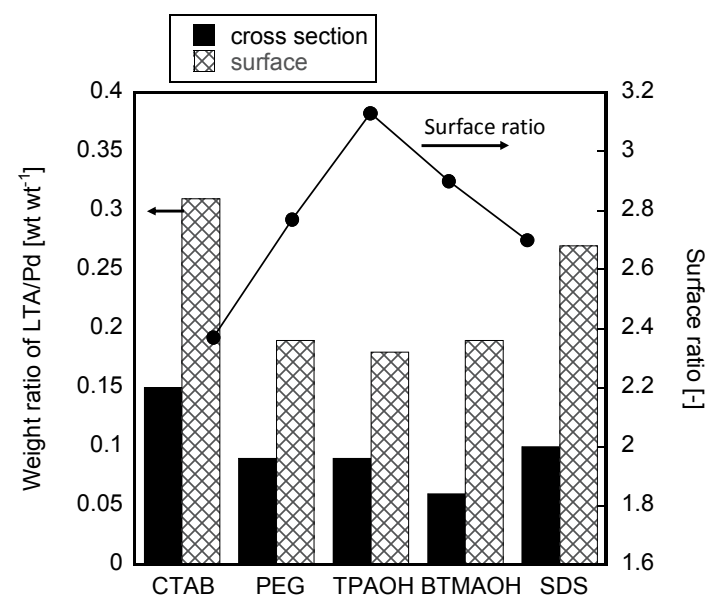

Fig. 5 Surface and cross-sectional LTA concentrations by changing the additives (CTAB, PEG, TPAOH,

BTMAOH and SDS) measured by EDX with surface ratio measured by AFM

The concentration in this experimental was slightly lower than that of CMC. Surface roughness obviously increased from the image shown in Fig. 2 (b). Effect of stirring was discussed elsewhere [4]. Viscosity of the solution increased from $0.52 \mathrm{cSt}$ to $0.64 \mathrm{cSt}$ by the $\mathrm{CTAB}$ addition. The effects of viscosity during the electroplating will be discussed in the following section.

LTA particles with about $1 \mu \mathrm{m}$ size were found from the surface view of Fig. 3 (cf. Fig. 2 (b)). However, LTA particles look physically attached on the rough surface instead of the co-deposition in the Pd film. In order to know the effects of CTAB concentration, CTAB concentration increased at $1.3 \times 10^{-2} \mathrm{~mol} \mathrm{~L}^{-1}$ that is one order larger than CMC. However, surface morphology and LTA concentration in the Pd film did not significantly changed by changing the CTAB concentration. So, cross-sectional observation should be conducted to confirm LTA co-deposition using the same surfactant concentration.

3.3 Effects of viscosity difference by changing additives

Fig. 4 shows SEM images of the surface morphologies of Pd films by changing additives (PEG, $\mathrm{TPAOH}$ and BTMAOH). Effect of viscosity change by changing the additives was discussed using PEG and CTAB. PEG is non-charged molecule. Thus, the viscosity of the electroplating solutions can be controlled without changing solution charge using PEG as an additive. Concentration of PEG was kept at $2.3 \mathrm{~g}$ $\mathrm{L}^{-1}$, and the viscosity was $0.64 \mathrm{cSt}$ that is the same to the conditions shown in Fig. 3. The difference between Fig. 3 and Fig4 (a) is the difference of charge of CTAB (+) and PEG $( \pm)$. These surface images look the similar to each other. Thus, effects of charge for the additives on the surface morphologies were not significant. LTA particles were also found from the both images.

TPAOH and BTMAOH were used as positive charge additives with low viscosity. These are quaternary ammoniums. TPAOH is used as an additive for disperse zeolite particles, such as LTA particles, in water. BTMAOH has phenyl group in the chemical formula. So, BTMAOH is a slightly hydrophobic chemical compared with TPAOH. Concentration for TPAOH and BTMAOH was kept at $1.4 \times 10^{-3} \mathrm{~mol} \mathrm{~L}^{-1}$ that is the same concentration for the CTAB solution. The SEM images shown in Fig. 4 (b), (c) are the similar to that shown in Fig. 3. The surface morphologies for TPAOH and BTMAOH expected to be smooth because of the low viscosity of the electroplating solutions. The plating mechanism for TPAOH and BTMAOH are not clear. Further discussions should be required for the consistent electroplating using both a quaternary ammonium and ceramic particles.

In conclusion for Fig.3 and Fig. 4, there are little effects on surface morphologies by the charge and viscosity of the additives. The roughness should be discussed by the point of current density $\left(0.20 \mathrm{~A} \mathrm{~cm}^{-2}\right)$ or so.

Fig. 5 shows surface ratios (measured surface area/ surface area before electroplating) and LTA concentrations from the surface and cross-section observations by changing the additives. The surface ratios are the similar between 2.4 and 3.1. These results are consistent with those shown in the surface observations (Fig.3, Fig. 4).

LTA concentrations in Pd flms were calculated from Si concentrations by EDX. There were two assumptions for the calculations. One was that LTA composition is $\mathrm{Na}_{12} \mathrm{Al}_{12} \mathrm{Si}_{12} \mathrm{O}_{48}$. The other was that all $\mathrm{Si}$ atoms in a Pd film derived from the LTA particles. 
All the LTA concentrations from the surface observations were higher than those from the cross-section observations. LTA particles were observed from all the surface images shown in Fig. 3 and Fig. 4. Thus, the higher LTA concentrations from the surface views are explained physically attached LTA particles. For the cross-sectional comparison, the LTA concentration for $\mathrm{CTAB}$ showed the maximum value $\left(0.15 \mathrm{wt} \mathrm{wt}^{-1}\right)$ among the 4 samples. CTAB is the best additive to obtain LTA particles in the Pd film prepared by the electroplating among PEG, TPAOH and BTMAOH. The LTA concentration for PEG was 0.09 wt $\mathrm{wt}^{-1}$. PEG is a neutral molecule, so there should be no effects on the electric plating without viscosity change for the solution. There was no LTA found from just adding LTA particles. There might be other effect to keep LTA particles in the electroplating solution by the high viscosity of the solution. The LTA concentration for TPAOH was $0.09 \mathrm{wt} \mathrm{wt}^{-1}$. The viscosity of TPAOH solution is almost the same to that of water. Thus, positive charge of TPAOH must be effective for the co-deposition. The LTA concentration for BTMAOH

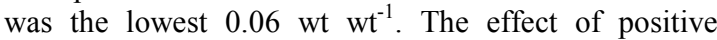
charge for BTMAOH should be lower than that for TPAOH.

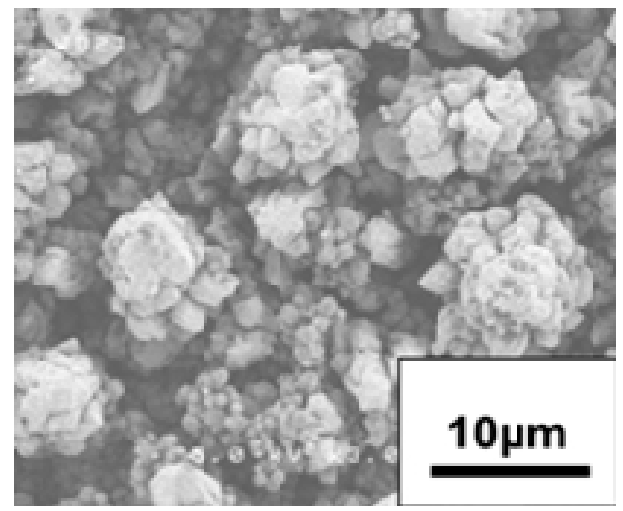

Fig. 6 SEM image for the surface of the sample with SDS (1.4 $\times 10^{-3} \mathrm{~mol} \mathrm{~L}^{-1}$, without stirring)

\subsection{SDS addition}

Finally, SDS addition was investigated. SDS is a negative charged surfactant. Fig. 6 shows a SEM image of the surface morphologies of Pd films by using SDS as an additive. The surface morphology looks the similar to those shown in Fig. 3 and Fig. 4. LTA particles were found from the figure.

Fig. 5 shows the surface and cross-sectional LTA concentrations for SDS addition with surface ratio. The data for CATB and PEG are also shown in the same figure. The surface ratio for SDS was 2.7 that was the same level among the other additives. LTA concentration from the surface observation was $0.27 \mathrm{wt}$ $\mathrm{wt}^{-1}$. This is slightly higher than that from the PEG sample. However, LTA concentration from the cross-sectional observation was $0.10 \mathrm{wt} \mathrm{wt}^{-1}$. This is the similar value with PEG and TPAOH. SDS is negative charged, so the coating of LTA particles by SDS must be negative effect on LTA concentration. SDS is also high viscosity additive. Thus, the effect of viscosity on the LTA concentration was dominant factor. We concluded that effect of SDS addition was not effective for co-deposition of Pd and LTA particles compared with $\mathrm{CTAB}$

\section{CONCLUSIONS}

Co-deposition of Pd and LTA ceramic particles were investigated by changing the additives to the electroplating solution. 5 types of additives were tested to know the effects of charge and viscosity of the additives. CTAB with positive charge and high viscosity showed the best performance to obtain high LTA concentration Pd thin layer. The LTA concentration was $0.15 \mathrm{wt} \mathrm{wt}^{-1}$ measured by the EDX. There was no LTA found from the samples by just adding LTA particles to the solution. Thus, the effect of CTAB was obvious. The surface morphologies for CTAB, PEG, TPAOH, BTMAOH and SDS look similar with the similar surface ratio (2.4-3.1). This is not explained by the former electroplating mechanism for the dendric structure, because both solution viscosity and charge of the additives were changed. The co-existence of LTA particle and the additives might affect the electroplating. However, further discussion should be required for the detailed mechanism.

\section{ACKNOLEDGEMENT}

Authors thank Mizusawa Ind. Chem. Co. for providing the LTA zeolite particles.

6. References

[1] S. Uemiya, Sep. Purif. Methods, 28(1), 51-85 (1999)

[2] Y. Zhang et al., J. Membr. Sci., 269, 60-65 (2006)

[3] S. Hara et al., J. Membr. Sci., 164, 289-294 (2000)

[4] M. Nomura et al., Bunri Gijutsu, 39(3), 46-52 (2009)

[5] T. Tubono et al., Proc. of 5th Conference of Aseanian Membrane Society, P3-22, Kobe, Japan (2009)

(Received Janurary 7, 2011; Accepted February 14, 2011) 